Bundesgesundheitsbl 2017 · 60:662-672

DOI 10.1007/s00103-017-2554-5

Online publiziert: 10. Mai 2017

(c) The Author(s) 2017. Dieser Artikel ist eine

Open-Access-Publikation.

CrossMark

Bund/Länder Ad-hoc Arbeitsgruppe,Gesundheitliche Anpassung an die Folgen
des Klimawandels (GAK)'
Berlin, Deutschland

\title{
Handlungsempfehlungen für die Erstellung von Hitzeaktionsplänen zum Schutz der menschlichen Gesundheit
}

angepasstes Risikoverhalten zu erreichen und präventive Handlungsmöglichkeiten zu etablieren [3, 4, 5]. Die Aktionspläne sollten sowohl Verhaltens- als auch verhältnispräventive Maßnahmen in allen Präventionsbereichen beinhalten.

$\mathrm{Da}$ es in Deutschland bislang keine harmonisierten Empfehlungen zu Aktionsplänen zur Hitzeprävention gibt, hat hierzu die Bund/Länder Ad-hoc AG GAK die vorliegenden allgemeinen Handlungsempfehlungen für die Erstellung von Hitzeaktionsplänen zum Schutz der menschlichen Gesundheit ausgearbeitet. Diese sind als einheitliche Grundlage für die konkrete Erarbeitung und Etablierung von auf die jeweilige Region abgestimmten, praktikablen Hitzeaktionsplänen zu verstehen. Sie richten sich in erster Linie an die Länder. Die Umsetzung erfolgt im Wesentlichen in den einzelnen Ländern auf kommunaler Ebene. Die Erarbeitung von Hitzeaktionsplänen kann zudem ein Beitrag zu einer übergreifenden Strategie zur Anpassung an den Klimawandel im Handlungsfeld menschliche Gesundheit sein.

In vielen Fällen sind Anpassungsmaßnahmen, welche vor Hitze schützen, ebenfalls zum Schutz vor erhöhter UVBelastung im Freien geeignet. Deshalb beinhalten diese Empfehlungen auch Maßnahmen zur Reduzierung der UVBelastung.

\section{Hintergrund}

Im Laufe des vergangenen Jahrhunderts stieg die mittlere Lufttemperatur in Deutschland um etwa $0,9^{\circ} \mathrm{C}$ an [1]. Wäh- rend mehrerer aufeinanderfolgender, mehrtägiger Hitzeereignisse, sogenannter „Hitzewellen“ in den Sommern 2003 und 2010 verstarben in Europa jeweils mehr als 40.000 Menschen zusätzlich aufgrund der Auswirkungen von ungünstigen meteorologischen Bedingungen (z. B. aufgrund extrem hoher Temperaturen) $[6,7,8,9]$. Allein in Deutschland forderte die Hitzeperiode im Jahr 2003 etwa 7000 Tote [7] sowie zahlreiche hitzebedingte Krankheitsfälle aufgrund von Dehydrierung, Hitzschlag sowie Herz- und Kreislauferkrankungen.

Erkrankungen aufgrund starker Hitze führen zu hohen Kosten, die das Gesundheitswesen und das Gemeinwohl stark belasten [10]. Für die Zeiträume 2021 bis 2050 bzw. 2071 bis 2100 wird eine weitere Zunahme der mittleren Jahrestemperatur für Deutschland um 0,5$1,5^{\circ} \mathrm{C}$ bzw. $1,5-3,5{ }^{\circ} \mathrm{C}$ mit einer erhöhten Häufigkeit von Hitzeperioden und einer damit einhergehenden Zunahme der hitzebedingten Todesfälle erwartet [1]. Schätzungen zufolge könnten Ende dieses Jahrhunderts bundesweit jährlich bis zu 8500 zusätzliche hitzebedingte Todesfälle eintreten [11].

Abhilfe kann hier - neben den weltweiten Bemühungen zur Bekämpfung des Klimawandels - nur durch koordinierte Aktivitäten zur Prävention von Hitzeauswirkungen auf die Bevölkerung geschaffen werden. Hierzu eignen sich Hitzeaktionspläne auf der Grundlage der Empfehlungen der Weltgesundheitsorganisation (WHO), die den Handlungsbedarf formulieren und Vorgaben machen, 


\section{Infobox 1 UV-Strahlung und Luftbelastung}

In den letzten Jahrzehnten haben Hautkrebserkrankungen um ein Vielfaches zugenommen. 2012 waren in Deutschland knapp 1,6 Millionen Menschen von einer Hautkrebs-Diagnose betroffen [14] - Tendenz weiter steigend. Jährlich versterben in Deutschland ca. 3000 Menschen an Hautkrebs [15]. Eine Ursache für diese hohen Hautkrebserkrankungszahlen liegt in der Exposition jedes Einzelnen mit UV-Strahlung. Risikofaktoren sind unter anderem Sonnenbrände (vor allem in der Kindheit), mit Unterbrechungen bzw. zeitlichen Zwischenräumen erfolgende hohe UV-Belastungen (z. B. Sonne bei Jahresurlauben auf nicht-adaptierte Haut) und die lebenslang erhaltene UV-Dosis [16]. Der Klimawandel bringt noch eine Verschärfung dieser Situation mit sich:

So wird davon ausgegangen, dass es als Folge der Klimaerwärmung zu einem veränderten Freizeitverhalten mit vermehrten Aktivitäten im Freien und einer dadurch erhöhten UV-Belastung kommen wird. Zudem treten im Frühjahr in Deutschland Niedrig-Ozon-Ereignisse in der Stratosphäre auf, durch die ein erhöhter UV-Strahlungsanteil bis zur Erdoberfläche gelangt. Je höher die UV-Exposition ist, desto höher wird das Hautkrebsrisiko.

Bodennahes Ozon wird bei intensiver Sonneneinstrahlung durch komplexe photochemische Prozesse aus Vorläuferschadstoffen - überwiegend Stickstoffoxiden und flüchtigen organischen Verbindungen - gebildet. Die gesundheitlichen Wirkungen von bodennahem Ozon bestehen in einer verminderten Lungenfunktion, entzündlichen Reaktionen in den Atemwegen und Atemwegsbeschwerden. Bei körperlicher Anstrengung, also bei erhöhtem Atemvolumen, können sich diese Auswirkungen verstärken. Empfindliche oder vorgeschädigte Personen, zum Beispiel an Asthma erkrankte Menschen, sind besonders anfällig.

Da Empfehlungen zum richtigen Verhalten bei Hitze in vielen Fällen ebenfalls eine Prävention gegenüber den gesundheitsschädlichen Wirkungen von bodennahem Ozon und seinen Vorläuferschadstoffen sowie gegenüber der erhöhten UV-Belastung bieten können, empfiehlt es sich, diese Belastungen hinsichtlich der Handlungsempfehlungen mit zu berücksichtigen.

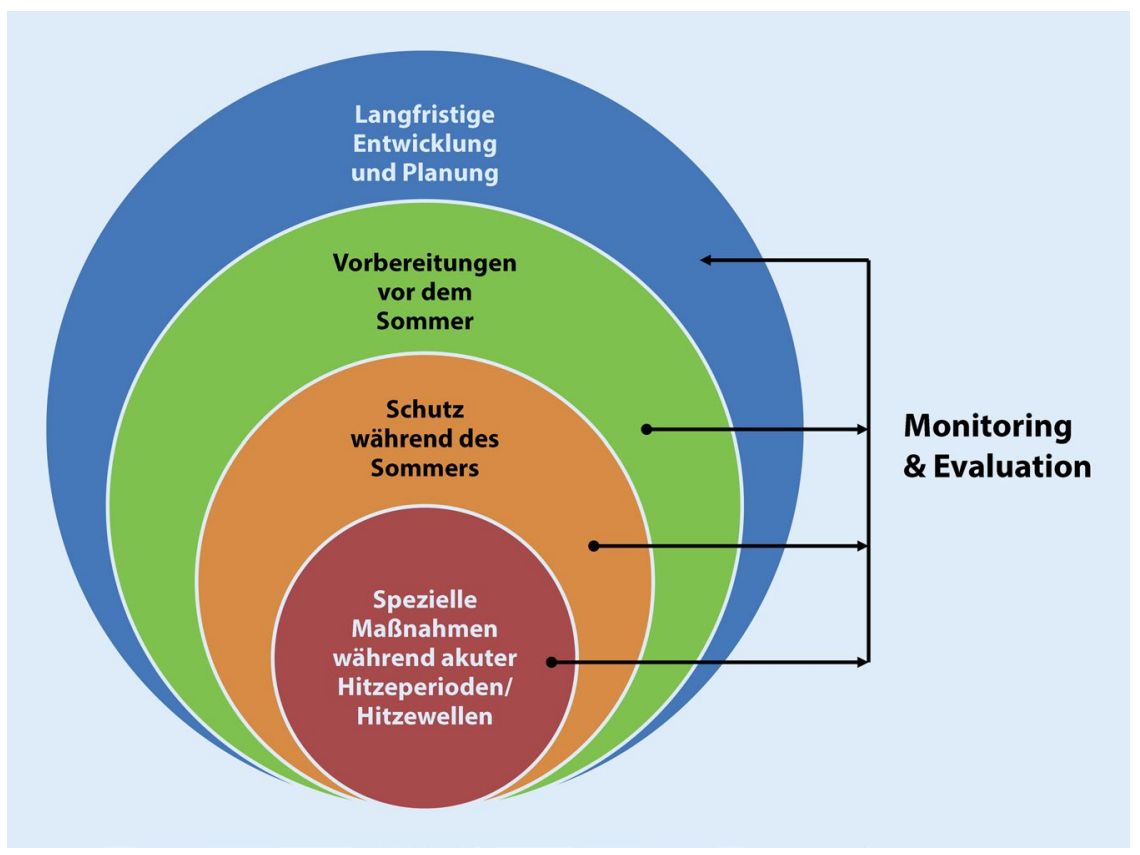

Abb. 1 S Schematische Darstellung der für die Umsetzung der Kernelemente eines Hitzeaktionsplans von der WHO vorgesehenen Zeithorizonte

auf welcher Ebene und von welcher Stelle welche Maßnahmen umgesetzt werden sollen. Konkrete Pläne sollten auf die jeweilige Region abgestimmt sein [12].

Ziel eines Hitzeaktionsplans ist, mittels Verhaltens- und verhältnispräventiver Maßnahmen die Hitze- und - soweit mit den gleichen Maßnahmen möglich - die UV-Exposition zu reduzieren, um hitze- und UV-bedingten Erkrankungen und möglichen Todesfällen vorzubeugen. Verhaltenspräventive Maßnahmen können von jeder einzelnen Person geleistet werden. Hierzu ist eine zielgruppenspezifische Aufklärung notwendig. Ergänzend sind verhältnispräventive Maßnahmen zu ergreifen, die Veränderungen des Lebensund Arbeitsumfeldes berücksichtigen [13].
Verhältnispräventive Ansätze zur Vorbeugung hitze- und UV-bedingter gesundheitlicher Folgen fehlen derzeit noch fast vollständig, obwohl sie leicht umsetzbar wären. $\mathrm{Zu}$ berücksichtigen sind hier vor allem vulnerable Personenkreise. Dazu zählen ältere Menschen, Pflegebedürftige und chronisch Kranke, die von einer Zunahme der Häufigkeit und Intensität extremer Hitzeereignisse besonders betroffen sind. Aber auch Säuglinge und Kleinkinder sind besonders anfällig. Auch Personen, die aufgrund von schwerer körperlicher Arbeit im Freien oder von Freizeitaktivitäten gegenüber Hitze und UV-Strahlung verstärkt exponiert sind, haben eine erhöhte Vulnerabilität gegenüber Hitze.

Neben der Belastung durch Hitze stellen auch die UV-Belastung sowie das Auftreten UV-Strahlungsassoziierter Luftverunreinigungen, wie bodennahes Ozon, gesundheitliche Probleme dar, die bei der Betrachtung von Anpassungsmaßnahmen relevant sind (•Infobox 1 ).

Die vorliegenden Handlungsempfehlungen für die Erstellung von Hitzeaktionsplänen zum Schutz der menschlichen Gesundheit in Deutschland basieren auf der von der Weltgesundheitsorganisation (WHO) veröffentlichten Leitlinie für die Erarbeitung eines Hitzeaktionsplans [12] und auf den Ergebnissen der Hessischen HEAT-Studie (durchgeführt von der Fachhochschule Fulda 2009-2012) [17].

Die Handlungsempfehlungen bestehen in Anlehnung an die WHO-Leitlinie aus acht Kernelementen, die in ihrer Reihenfolge nicht zwingend nacheinander folgend bzw. aufeinander aufbauend zu verstehen sind.

Die acht Kernelemente sind:

I. Zentrale Koordinierung und interdisziplinäre Zusammenarbeit

II. Nutzung eines Hitzewarnsystems

III. Information und Kommunikation

IV. Reduzierung von Hitze in Innenräumen

V. Besondere Beachtung von Risikogruppen

VI. Vorbereitung der Gesundheitsund Sozialsysteme

VII. Langfristige Stadtplanung und Bauwesen

VIII. Monitoring und Evaluation der Maßnahmen 


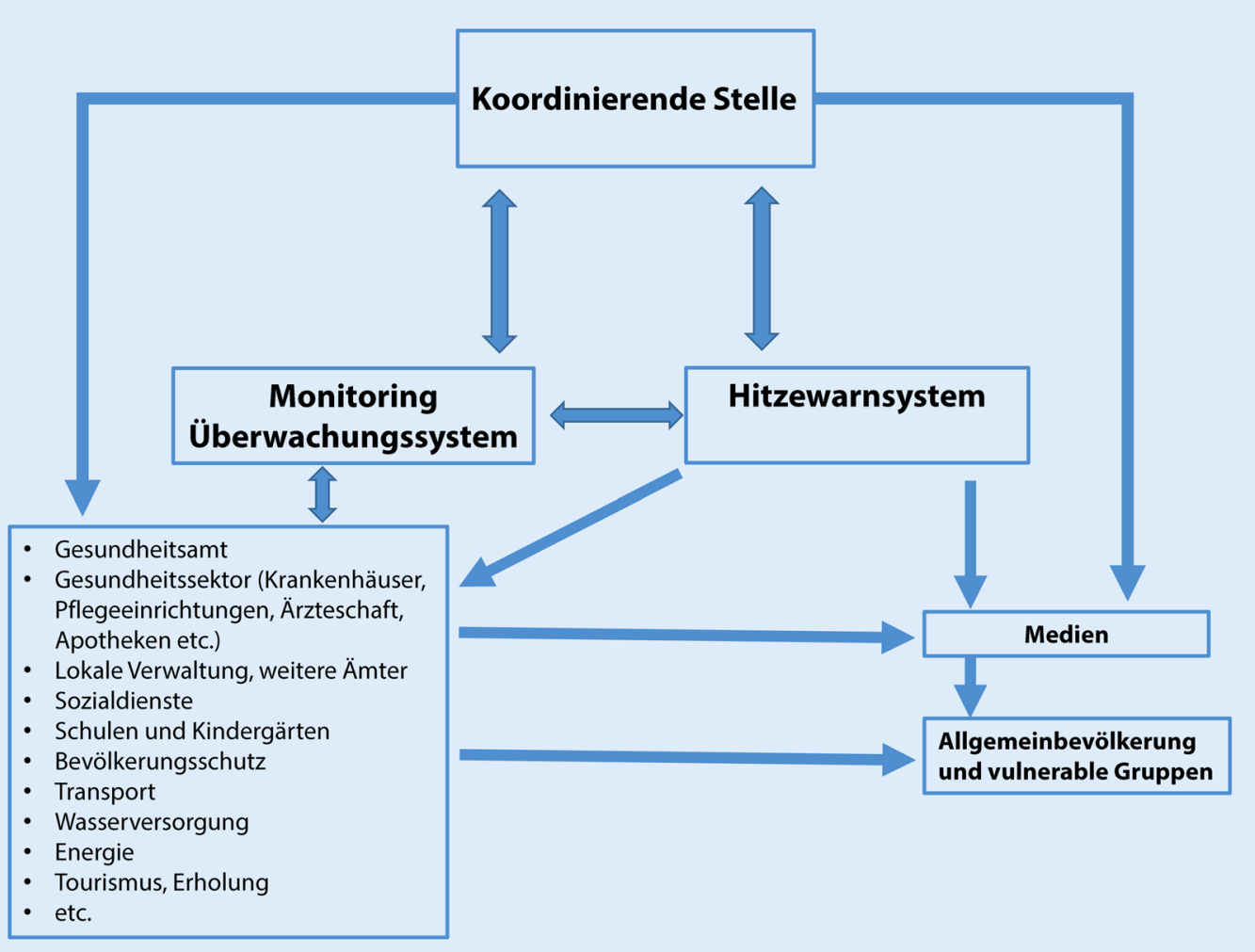

Abb. $2 \triangleleft$ Möglicher Informationsfluss zwischen einer koordinierenden Stelle und anderen Beteiligten in Hitzeaktionsplänen (nach Matthies et al. 2008, Abb. 2, S. 9 [12])
Diese Handlungsempfehlungen beinhalten sowohl kurzfristige, ohne große Investitionen umsetzbare Sofortmaßnahmen als auch langfristige Maßnahmen, die bei einer Planung grundsätzlich berücksichtigt werden sollten. Für die Umsetzung der vorgenannten acht Kernelemente empfiehlt die WHO ein Vorgehen in fünf Zeithorizonten [12]:

- Langfristige Entwicklung und Planung

- Vorbereitungen rechtzeitig vor dem Sommer

- Schutz während des Sommers

- Spezielle Maßnahmen während akuter Hitzeperioden/Hitzewellen

- Monitoring und Evaluation

Diese sind in der nachfolgenden $\bullet$ Abb. 1 schematisch dargestellt.

Die Organisation, Zusammenarbeit und Kommunikation von Informationen zwischen einer koordinierenden Stelle/Institution und den eingebundenen Mitwirkenden eines Hitzeaktionsplans kann gemäß den in - Abb. 2 dargestellten, von der WHO vorgeschlagenen Informationswegen erfolgen.

\section{Handlungsempfehlung zu Kernelement I}

\section{Zentrale Koordinierung und interdisziplinäre Zusammenarbeit}

Laut WHO ist die Verständigung auf eine Leitungsinstitution sinnvoll. Vor dem Hintergrund der in Deutschland dezentral bzw. föderal geteilten Zuständigkeiten wird die Einrichtung einer zentralen Koordinierungsstelle empfohlen. Diese hat die Koordinierungsfunktion und behördenübergreifende Zuständigkeit, um in $\mathrm{Zu}$ sammenarbeit mit weiteren Behörden und Einrichtungen unterschiedlicher Zuständigkeit einen Hitzeaktionsplan in Kommunen einzuführen. Sie hat die Aufgabe, kurz-bis langfristige Maßnahmen einzuleiten.

1. Eine zentrale Koordinierungsstelle sollte auf Landesebene bspw. in einer Gesundheitsbehörde eingerichtet werden. Je nach Dauer und Ausprägung der Hitzesituation könnte im akuten Fall die Koordination auch von einer anderen Behörde ausgeübt werden.

2. Die zentrale Koordinierungsstelle soll die behördenübergreifende Zuständigkeit zur Koordinierung der Zu- sammenarbeit von einzubeziehenden Behörden und Einrichtungen haben (analog einem Krisenmanagement im Katastrophenfall).

3. Es wird empfohlen, dass sich die zentralen Koordinierungsstellen der Länder austauschen.

4. Die Aufgaben der zentralen Koordinierungsstelle sind im Einzelnen:

- Einrichtung eines zentralen Netzwerks aller Beteiligten, die mit der Entwicklung von Hitzeaktionsplänen befasst sind (z. B. Landesgesundheitsbehörden, kommunale Landesverbände, kassenärztliche Verbände, Landesärztekammern, Träger öffentlicher Einrichtungen).

- Gemeinsam mit dem zentralen Netzwerk identifiziert die zentrale Koordinierungsstelle die relevanten Institutionen (staatliche und nicht-staatliche), die vor Ort in den jeweiligen Kommunen Maßnahmen umsetzen können. Beispiele sind Feuerwehren, Not- und Rettungsdienste, Krankenhäuser, Ärzteschaft/Praxen, Apothekerschaft, ambulante und stationäre Pflegeeinrichtungen, Einrichtungen zur 
Rehabilitation, Kindergärten, Schulen, Hilfsorganisationen, Behindertenhilfen und Heimaufsichten.

- Zur genaueren Situationsanalyse und Planung der konkreten Maßnahmen können weitere Organisationen sowie interdisziplinäre Fachkräfte hinzugezogen werden, z. B. aus den Gebieten Gesundheits-, Pflege-, Ernährungs- und Sozialwissenschaften, Medizin, Gesundheitsingenieurwesen sowie Medizinischer Dienst und Krankenversicherungen.

5. Aufgaben des zentralen Netzwerks:

- Es unterrichtet die Kommunen als dezentrale Koordinierungsstellen über die zur Einbindung empfohlenen Institutionen. Die Festlegung der Zuständigkeiten in den einzubeziehenden Einrichtungen vor Ort sowie die Planung konkreter Maßnahmen und deren zeitliche Umsetzung auf Basis der Handlungsempfehlungen zu den Kernelementen II bis VIII obliegt den Mitwirkenden auf kommunaler Ebene, die hierfür ein dezentrales Netzwerk bilden. Die Festlegungen werden der Koordinierungsstelle rückgemeldet.

- Als notwendig erachtete übergeordnete Maßnahmen, die auf kommunaler Ebene nicht umsetzbar sind (bspw. Regionalplanung), sollten vom zentralen Netzwerk in direktem Kontakt mit den relevanten Institutionen geplant werden.

6. Nach der Umsetzung von Maßnahmen - insbesondere nach akuten Hitzeereignissen - evaluieren die zentrale Koordinierungsstelle, das zentrale Netzwerk sowie kommunale Mitwirkende die getroffenen Maßnahmen und Abläufe und passen daraufhin die Koordination, Empfehlungen und deren Umsetzung an (siehe auch Handlungsempfehlungen zu Kernelement VIII ,Monitoring und Evaluation der Maßnahmen').

Zur Verdeutlichung der Ebenen der Koordinierung und der interdisziplinären Zusammenarbeit siehe nachfolgende - Abb. 3.

\section{Handlungsempfehlung zu Kernelement II}

\section{Nutzung des Hitzewarnsystems}

Die WHO empfiehlt die Verständigung auf die Nutzung eines verlässlichen Hitzewarnsystems, das die Dauer des bevorstehenden Hitzeereignisses, Verhaltensempfehlungen sowie die gesundheitlichen Risiken kommuniziert.

Das Hitzewarnsystem wird vom Deutschen Wetterdienst (DWD) betrieben und ist in Deutschland seit mehr als zehn Jahren etabliert. Der DWD gibt die für Deutschland geltenden Hitzewarnungen aus.

Hitzewarnungen richten sich an zuständige Stellen und sind insbesondere für Alten- und Pflegeheime sowie Kindergärten vorgesehen. Sie richten sich aber auch an andere Einrichtungen, z. B. ambulante und stationäre Pflegedienste sowie an die Allgemeinbevölkerung. Die Warnungen werden landkreisbezogen herausgegeben.

1. Es wird empfohlen, Hitzeaktionspläne auf Basis des Hitzewarnsystems des DWD auszuarbeiten. Der DWD gibt Hitzewarnungen heraus, wenn eine starke Wärmebelastung für mindestens 2 Tage in Folge vorhergesagt wird und eine ausreichende nächtliche Auskühlung der Wohnräume nicht mehr gewährleistet ist. Es gibt zwei Warnstufen [18]:

Hitzewarnstufe I: „starke Wärmebelastung" („Gefühlte Temperatur" an zwei Tagen in Folge über $32^{\circ} \mathrm{C}$, zusätzlich nur geringe nächtliche Abkühlung); Hitzewarnstufe II: „extreme Wärmebelastung "(Gefühlte Temperatur" über $38^{\circ} \mathrm{C}$ am frühen Nachmittag). Die Warnungen gelten grundsätzlich für einen ganzen Tag und damit auch für die Nachtsituation. Die Hitzewarnungen werden vom DWD per Newsletter (www.dwd.de/newsletter), über die Internetseite des DWD oder über Apps (erhältlich in den üblichen AppStores) veröffentlicht.

Sinnvoll ist auch der gleichzeitige Bezug weiterer hitzeassoziierter gesundheitsrelevanter Meldungen:

- Über herrschende UV-Bestrahlungsstärken (UV-Index) informieren das Bundesamt für Strahlen- schutz (BfS; www.bfs.de/uv-index) und der DWD (www.dwd.de/uvindex)

- Informationen über UV-Bestrahlungsstärken werden vom BfS und vom DWD auch per Newsletter (www.bfs.de/uv-newsletter bzw. www.dwd.de/newsletter) oder über Apps (erhältlich in den üblichen App-Stores) veröffentlicht.

- Informationen über bodennahes Ozon von den zuständigen Länderbehörden (meist Landesumweltämter); Verbreitung z. B. über Rundfunk und Fernsehen

Auch die Informationsdienste/Apps des Bevölkerungsschutzes wie z. B. ,Katwarn' oder ,Nina' informieren über wetterassoziierte Extremsituationen.

2. Die Behörden und Organisationen, die laut des Hitzeaktionsplans aktiv Maßnahmen einleiten, sollen angehalten werden, den Hitzewarn-Newsletter des DWD zu abonnieren. Dies sollten mindestens folgende Einrichtungen vornehmen:

- Gesundheitsministerien der Länder

- Gesundheitsämter der Kommunen

- Verbände und Einrichtungen, die in der gesundheitlichen und sozialen Versorgung der Bevölkerung tätig sind (z. B. Schulen, Kindergärten, Rettungsdienste, Krankenhäuser sowie Pflegedienste und -einrichtungen)

- Soziale Netzwerke und Nachbarschaftshilfen zur Verbreitung der Hitzewarnungen

3. Es wird empfohlen, als Reaktion auf Hitzewarnstufe I kurzfristig anzuwendende Maßnahmen (s. Handlungsempfehlungen zu Kernelement IV) über die etablierte Kommunikationskaskade (s. Handlungsempfehlungen zu Kernelement III) innerhalb der relevanten staatlichen und nicht-staatlichen Einrichtungen, die Maßnahmen vor Ort umzusetzen haben (s. Handlungsempfehlungen zu Kernelement I), zu kommunizieren. Als Reaktion auf Hitzewarnstufe II ist es empfehlenswert, die Warnung vor einem prognostizierten Extremhitzeereignis und kurzfristig anzuwendende Maßnahmen zusätzlich über Massenmedi- 


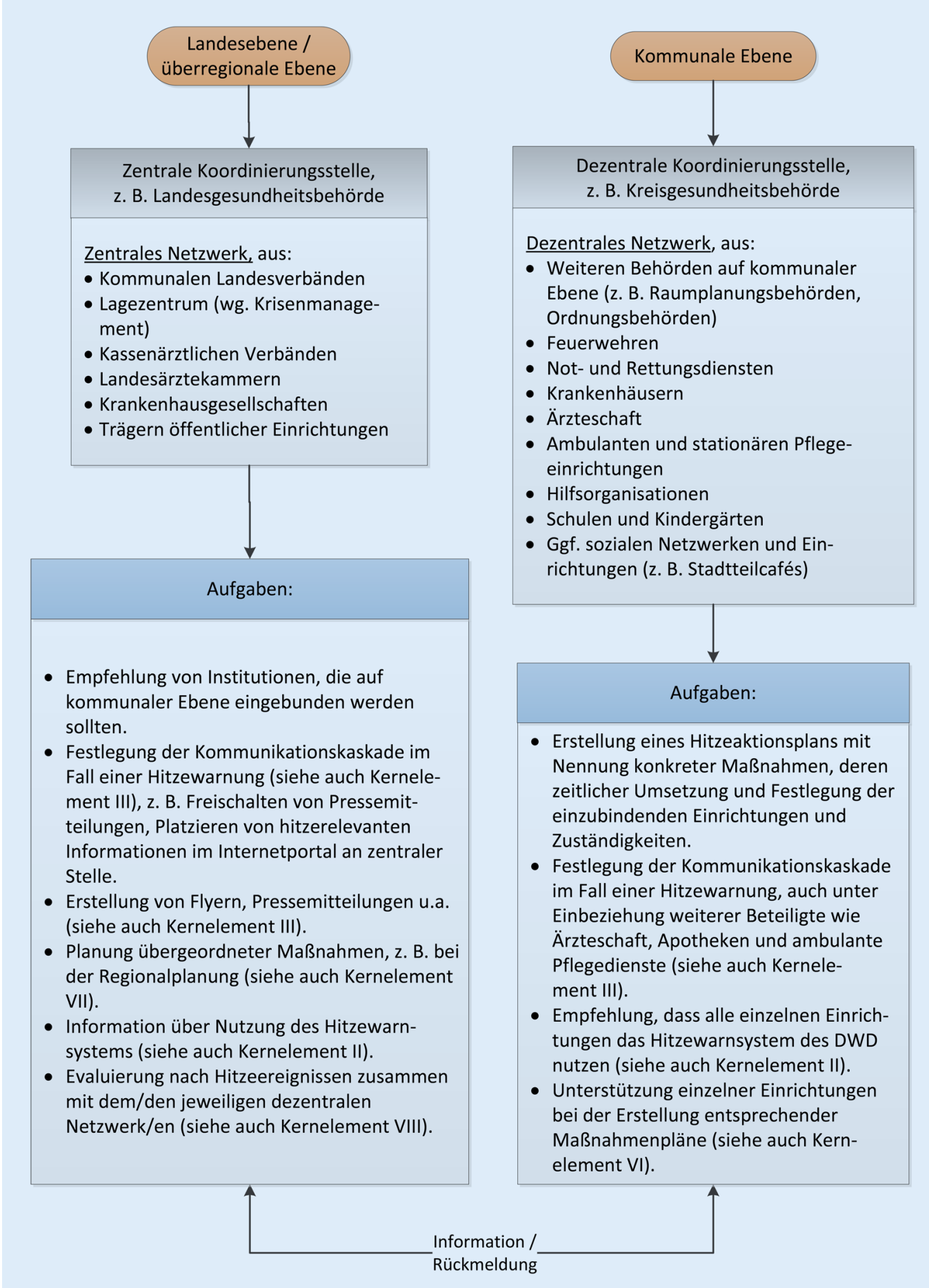

Abb. $3<$ Skizze zur zentralen Koordinierung und zur interdisziplinären Zusammenarbeit (Kernelement I mit teilweiser Berücksichtigung weiterer Kernelemente) 
en wie Rundfunk und Fernsehen aktiv zu kommunizieren.

\section{Handlungsempfehlung zu Kernelement III}

\section{Information und Kommunikation}

Die WHO empfiehlt die Erstellung und Kommunikation von hitzebezogenen Gesundheitsinformationen für die Bevölkerung (wer kommuniziert was, wann und wie?).

Bei der Kommunikation von hitzeassoziierten Gesundheitsinformationen sind die vorausschauende Planung sowie der Akutfall eines unmittelbar bevorstehenden Hitzeereignisses getrennt zu berücksichtigen.

Des Weiteren sind Informationspakete sind für verschiedene Zielgruppen $\mathrm{zu}$ erarbeiten.

\section{Vorausschauende Planung}

1. Die Kommunikationsinhalte sowie die Kommunikationswege sollten unabhängig vom Vorliegen einer Hitzesituation festgelegt werden (analog der Netzwerkstruktur, siehe Handlungsempfehlung zu Kernelement I). Es ist erforderlich, die Zuständigkeit und Verantwortlichkeit für die Kommunikation festzulegen (Kommunikationskaskade), idealerweise in Form eines Ablaufplans im Fall einer DWD-Hitzewarnung (vergleichbar mit einem Alarmplan).

2. Zentrale Fragestellungen hierbei sind:

Was soll kommuniziert werden? Die gesundheitlichen Präventionsempfehlungen sollten möglichst zielgruppenspezifisch ausgearbeitet werden.

Beispiele für grundlegende Informationen:

- Internetseite des DWD http://www.dwd.de/DE/leistungen/ hitzewarnung/hitzewarnung.html

- Publikation UBA und DWD Ratgeber: Klimawandel und Gesundheit. Informationen zu gesundheitlichen Auswirkungen sommerlicher Hitze, Hitzewellen und Tipps zum vorbeugenden Gesundheitsschutz https://www.umweltbundesamt.de/ publikationen/ratgeber-klimawandel-gesundheit

- Publikation „Hitzeknigge“ des UBA https://www.umweltbundesamt.de/ sites/default/files/medien/364/dokumente/schattenspender_hitzeknigge.pdf

Wie soll kommuniziert werden? Es kommen verschiedene Möglichkeiten in Frage: Zeitungen, Fernsehen, Radio, soziale Medien sowie weitere Multiplikatorengruppen. Damit können bestimmte Bevölkerungsgruppen wie ältere Menschen und Jüngere gezielt angesprochen werden.

\section{Wann soll kommuniziert werden?}

Es sollte genau festgelegt sein, in welchem Zeitabstand nach einer erfolgten DWD-Hitzewarnung die Informationen durch die o.g. Medien kommuniziert werden sollen. Da in der Regel nur ein kurzer Handlungsspielraum zur Verfügung steht, ist es notwendig, vorab formulierte Presseinformationen (evtl. auch Interviews oder andere Medienbeiträge wie das Video „Hitzewarnsystem des DWD“ auf YouTube) bereit zu halten oder darauf zu verlinken.

3. Auf den Internetseiten der Länder und Kommunen sollten dauerhaft Informationen angeboten werden, die sich mit Vorsorgemaßnahmen bezüglich der Anpassung an Hitzesituationen befassen. Auch Informationsbroschüren sollten unabhängig von der konkreten Gefährdungssituation vorgehalten werden und im Internet als Download verfügbar sein.

4. Die verschiedenen Informationsangebote von Bund, Ländern, Kommunen, Krankenversicherungen usw. sollten aufeinander abgestimmt sein.

5. Jahreszeitlich aktuelle Beiträge z.B. in Apothekenzeitschriften sind eine Möglichkeit, besonders ältere Menschen zu erreichen. Des Weiteren kann es für einige Personengruppen vorteilhaft sein, wenn zusätzlich telefonische Beratung angeboten wird (analog dem Hitzetelefon „Sonnenschirm" des Gesundheitsamtes der Region Kassel).
6. Fremdsprachliche Hinweise sollten bereitgehalten werden (z. B. für Personen mit Migrationshintergrund, Touristen).

\section{Kommunikation im Akutfall eines Hitzeereignisses}

1. Bei Bevorstehen oder Vorliegen eines Hitzeereignisses können verhaltensbezogene Informationen auch im persönlichen Gespräch von Ärzten und Ärztinnen an Patientinnen und Patienten weitergegeben werden. In Flyern/Broschüren oder TV-Spots in Praxiswartezimmern oder in Apotheken (Verteilung über die Ärzte- bzw. Apothekenkammern) kann auf die gesundheitlichen Risiken und Maßnahmen zum Schutz hingewiesen werden. Auch Angehörige von allein lebenden älteren Personen oder Personen, die in der Handlungsempfehlung zu Kernelement $\mathrm{V}$ aufgeführt sind, sollten in die Informationsweitergabe eingebunden werden.

2. Kindergärten, Schulen, Krankenhäuser und Alten- sowie Pflegeheime sollten im akuten Fall aktiv benachrichtigt und mit Informationsmaterial versorgt werden.

Auch das deutsche Hotel- und Gaststättengewerbe sollte zur Information von Reisenden über drohende Hitze und Anpassungsmöglichkeiten in Kenntnis gesetzt werden.

\section{Handlungsempfehlung zu Kernelement IV}

\section{Reduzierung von Hitze in Innenräumen}

Die WHO empfiehlt die Etablierung von Maßnahmen zur Reduzierung von Hitze in Innenräumen (kurz- und mittelfristige Maßnahmen, um die Innenraumtemperaturen während starker und extremer Hitze zu mindern).

Die Vermeidung des Aufheizens von Innenräumen ist besonders bedeutsam. Die zu empfehlenden Maßnahmen sollten den entsprechenden Zielgruppen (z.B. Personen, die die Gebäude/Räumlichkeiten planen oder nutzen) kommuniziert werden. Die mittelfristigen Maßnah- 
men bedürfen einer baulichen Anpassung oder sogar einer längerfristigen Planung. Langfristige Maßnahmen finden sich unter Handlungsempfehlung VII.

1. Kurzfristige Maßnahmen - Empfehlungen zum Verhalten sowie einfache technische Möglichkeiten:

- Insbesondere bei direkter Sonneneinstrahlung ist ein Verdunkeln/ Verschatten durch Vorhänge, Jalousien, Markisen, Sonnenschirme, Fenster- oder Rollläden zu empfehlen.

- Innerhalb eines Hauses/einer Wohnung sollten kühle Räume aufgesucht werden.

- Ventilatoren können eingesetzt werden, falls dies vertragen oder als angenehm empfunden wird.

- Das Lüftungsverhalten sollte im Fall ausgeprägter Hitze angepasst werden:

So ist es zweckmäßig, nur während der kühleren Nacht- und Morgenstunden die Fenster zu öffnen und zu lüften. Je nach Situation des Umfeldes ist es sinnvoll, vor den entsprechenden Fenstern Mückenschutzgitter anzubringen.

- Wärme abgebende Geräte sollten möglichst nicht verwendet werden, da sie zu einer zusätzlichen Wärmebelastung führen können.

2. Mittelfristige Maßnahmen - Gebäude bezogene Kühlungsmaßnahmen:

- Installation von Rollläden, Außenjalousien, Blendläden mit Luftschlitzen, Markisen und Sonnensegeln

- Wand- und Dachisolierungen schützen nicht nur im Sommer vor dem Aufheizen der Gebäude, sondern im Winter auch vor der Auskühlung der Räumlichkeiten.

- Mikroklimatische Abkühlungseffekte können für Innenräume auch durch Dach- und Fassadenbegrünung sowie durch Laubbäume an Straßen, in Gärten und Grünanlagen erzielt werden.

- Der Einbau technischer Kühlverfahren (auch Klimaanlagen) sollte bei jeder Renovierung z. B. von Krankenhäusern, Alten- und Pflegeheimen in Betracht gezogen werden.
3. Aktive Kühlung von Innenräumen:

- Klimaanlagen (auch mobile Geräte) für den privaten Bereich sind nur dann zu empfehlen, wenn andere Maßnahmen nicht zum Erfolg führen und alle baulichen und technischen Möglichkeiten ausgeschöpft sind. Durch ihren hohen Stromverbrauch und die Abgabe von Wärme an die Außenluft tragen sie zum Klimawandel und zu einer weiteren Aufheizung der allgemeinen Atmosphäre im Außenbereich vor allem in Städten (Wärmeinseleffekt) bei.

\section{Handlungsempfehlung zu Kernelement V}

\section{Besondere Beachtung von Risikogruppen}

Die WHO empfiehlt die besondere Beachtung von Risikogruppen, die bei Hitzeereignissen besonders gefährdet oder besonders schutzbedürftig sind.

Bestimmte Risikopersonen bzw. -gruppen (vulnerable Personengruppen) müssen bei der Auswahl von geeigneten Maßnahmen besonders berücksichtigt werden. Auch hinsichtlich einer geeigneten Herangehensweise bei der Kommunikation von Hitzerisiken für die Gesundheit bestehen Besonderheiten. Dies ist vor allem wichtig vor dem Hintergrund einer älter werdenden Gesellschaft.

Insbesondere bei mehreren aufeinanderfolgenden Hitzetagen (einer sogenannten Hitzewelle) ist durch die Hitzebelastung mit einer Verstärkung von negativen Folgen, zum Beispiel für das Herz-Kreislaufsystem und die Nieren, zu rechnen. In der Regel sollten Personen, die zu den vulnerablen Gruppen zählen, vor allem auf eine verstärkte Flüssigkeitszufuhr achten bzw. sollte ihnen eine höhere Flüssigkeitsmenge als bei normalen Temperaturen zugeführt werden. Evtl. ist eine Anpassung der Medikation (insbesondere von Diuretika) vorzunehmen. Zudem sollten vulnerable Personen während eines Hitzeereignisses angemessen gekleidet sein (luftige Kleidung, die bei Sonnenexposition einen ausreichenden UV-Schutz gewährleistet) und sich möglichst wenig im Freien aufhalten, sondern eher in kühleren Innen- räumen (vgl. auch Handlungsempfehlungen zu Kernelement VI).

1. Folgende Risikogruppen bedürfen besonderer Beachtung:

- Ältere Menschen

In der Regel liegt bei dieser Gruppe eine reduzierte Fähigkeit des Körpers zur Hitzeregulation vor und das Durstgefühl nimmt mit zunehmendem Alter ab.

- Isoliert lebende Menschen Insbesondere bei isoliert lebenden älteren Menschen ist das Risiko für gesundheitliche Hitzeauswirkungen groß, weil eine soziale Kontrolle und Hilfsangebote fehlen.

- Pflegebedürftige Menschen Bei diesen Personen bestehen oft gesundheitlich bedingte Beeinträchtigungen, die deren Handlungsmöglichkeiten stark einschränken. Ein zusätzliches Risiko kann durch bestimmte Erkrankungen im Einzelfall vorliegen.

- Personen mit starkem Übergewicht Sie reagieren auf Hitzebelastungen besonders intensiv, weil der Organismus dann neben der ohnehin bestehenden gesundheitlichen Grundbelastung einer weiteren außergewöhnlichen Belastung ausgesetzt ist und z. B. das Herz-Kreislaufsystem kaum Reservekapazitäten aufweist.

- Menschen mit chronischen Erkrankungen

Der Organismus dieser Menschen ist oft aufgrund seines Gesundheitszustandes nicht in dem Maße in der Lage, auf extreme Situationen zu reagieren wie es bei Gesunden der Fall ist. Außerdem kommt es bei chronischen Erkrankungen in extremen Situationen oftmals zu einer Verschlechterung der Symptomatik.

- Menschen mit fieberhaften Erkrankungen

Bei fieberhaften Erkrankungen ist es wichtig, dass der Körper Hitze abgeben kann. Daher ist eine deutliche Erhöhung der Raumtemperatur des Krankenzimmers hier besonders problematisch und sollte durch entsprechende Maßnahmen vermieden werden. 
- Menschen mit Demenz

Bei Menschen mit Demenz liegt

eine reduzierte Möglichkeit vor, auf extreme Situationen adäquat zu reagieren. Insbesondere die Aufnahme von ausreichend Flüssigkeit ist bei Menschen mit Demenzerkrankungen ohne Betreuung häufig nicht gewährleistet.

- Menschen, die bestimmte Medikamente einnehmen

Die Wirkungen und Nebenwirkungen von Medikamenten (insb. Diuretika und blutdrucksenkende Mittel) können bei Hitzeeinfluss verändert sein. In einigen Fällen wird eine Anpassung der Medikation erforderlich sein, um Dekompensation zu vermeiden.

- Personen, die thermophysiologische Anpassungsprobleme haben Nicht alle Menschen vertragen Hitze gleich gut. Bei manchen Menschen führt eine starke Hitzebelastung beispielsweise zu Kreislaufproblemen aufgrund von Blutdruckabfall.

- Säuglinge und Kleinkinder Hier besteht eine besondere Schutzbedürftigkeit, zum einen weil eine besondere Empfindlichkeit besteht, aber auch weil hier eine ausgeprägte Abhängigkeit von den Aufsichtspersonen gegeben ist. Ohne Anleitung ist bei Kindern keine Einsicht oder sinnvolles Handeln im Hinblick auf Hitze- und UV-Schutz zu erwarten.

2. Berücksichtigung finden sollten außerdem Personen, die im Freien körperlich intensiv tätig sind (z. B. Berufe des Baugewerbes, der Landwirtschaft und Gastronomie). Obgleich sie keine der typischen Risikogruppen darstellen, bedürfen diese Personen aufgrund ihrer oft extremen Hitze- und UV-Exposition besonderer Aufmerksamkeit und einer Anpassung der Arbeitsbedingungen durch den Arbeitgeber (z. B. durch Anpassung der Arbeitszeit und Pausenregelung).

3. Obdachlose bedürfen in Extremsituationen oftmals einer individuellen Betreuung durch die Sozialdienste.

\section{Handlungsempfehlung zu Kernelement VI}

\section{Vorbereitung der Gesundheits- und Sozialsysteme}

Die WHO empfiehlt die Vorbereitung des Gesundheits- und Sozialsystems (u.a. durch Fort- und Weiterbildung des Medizin- und Pflegepersonals).

Bei der Vermeidung von gesundheitlichen Auswirkungen von Hitzeereignissen kommt den Beteiligten des Gesundheits- und Sozialwesens eine zentrale Bedeutung $\mathrm{zu}$, da diese eine direkte Schnittstelle zu den Hauptrisikogruppen, insbesondere den älteren und kranken Menschen, darstellen. Dies ist vor dem Hintergrund einer zunehmend älter werdenden Gesellschaft von besonderer Bedeutung.

Fort- und Weiterbildung von Beschäftigten im Gesundheits- und Sozialwesen kann dazu beitragen, wichtige Inhalte zum adäquaten Handeln während Hitzeperioden $z u$ vermitteln. Es ist zu prüfen, welche Berufsgruppen über Fort- und Weiterbildungsmaßnahmen geschult werden sollten und wie die Schulungen das bereitgestellte Informationsmaterial sinnvoll ergänzen.

Maßnahmenpläne für Einrichtungen zur Vorbereitung auf Hitzeereignisse sollten insbesondere entwickelt werden für:

- Alten- und Pflegeheime

- Einrichtungen für Menschen mit körperlichen und geistigen Einschränkungen

- Krankenhäuser, Not- und Rettungsdienste

- Einrichtungen zur Rehabilitation

- Schulen

- Kindertageseinrichtungen

- bestimmte Arbeitsstätten

Ein Beispiel für Einrichtungen der stationären Pflege sind die Handlungsempfehlungen der hessischen Heimaufsicht (siehe $\bullet$ Infobox 2 und Weiterführende Literatur).

Konkrete Pflege- und Betreuungsmaßnahmen auch in der ambulanten Pflege können beispielsweise sein:

- Anpassung und Überwachung des Trinkverhaltens

- Anpassung der Ernährung
- Anpassung der Kleidung, z. B. bei pflegebedürftigen Personen

- Anpassung der Medikation

- Tageszeitliche Anpassung der Aufenthalte an beschatteten Plätzen im Freien bzw. in kühlen Räumen

- Anpassung der Freizeitaktivitäten

Hitzebedingte Auswirkungen auf Infrastrukturen können auch Auswirkungen auf die Versorgung haben. Es ist wichtig, an mögliche Nebeneffekte wie Wasserknappheit und Ausfälle im Bereich der Stromversorgung zu denken. Bei großer Hitze ist außerdem eine intakte Kühlkette in der Lebensmittelversorgung evtl. nicht mehr gewährleistet.

Es sollte geprüft werden, ob ärztliche Behandlungsmaßnahmen anzupassen sind.

Mögliche Hitzeereignisse sollten frühzeitig im Rahmen des Personalkräfteeinsatzes sowie der Urlaubsregelung im Gesundheitswesen in der Sommerzeit berücksichtigt werden. Bei akuter Hitze können Anpassungen der Personalausstattung erforderlich sein.

Besonders in Einrichtungen des Gesundheitswesens sollten kühle Räume zur Verfügung gestellt werden. Dafür ist es erforderlich, solche Bereiche frühzeitig $\mathrm{zu}$ identifizieren und als solche in den Maßnahmenplänen auszuweisen.

In Einrichtungen des Gesundheitswesens sind ggf. bauliche Maßnahmen zum Schutz vor Hitze in Angriff zu nehmen (Verschattungen, Raumventilatoren, ggf. Klimaanlagen). Über diese Maßnahmen informieren die Handlungsempfehlungen IV und VII.

\section{Handlungsempfehlung zu Kernelement VII}

\section{Langfristige Stadtplanung und Bauwesen}

Die WHO empfiehlt die langfristig ausgerichtete Stadtplanung zur Reduzierung der Hitzebelastung im Bau-, Energie- und Transportsektor.

Bei der Planung und Umsetzung von bau- und stadtplanerischen Maßnahmen ist Augenmaß erforderlich. Es bedarf der Berücksichtigung der ganzjährigen, regionalspezifischen und klimatischen Situa- 
tion. So kommen manche Maßnahmen in Gegenden Deutschlands, die ohnehin kühler sind, möglicherweise nicht oder in anderer Form in Frage. Langfristige und aufwendige Anpassungsmaßnahmen im Bereich Hitzeschutz stellen immer einen Abwägungsprozess unter Berücksichtigung anderer Belange dar und sollten regionaldifferenziert betrachtet werden.

Gebäudebezogene Maßnahmen:

- Vorgaben entwickeln für den Hitzeschutz von Gebäuden (z. B. Thermoglas, in Fenster integrierte Lamellenjalousien, Beschattung durch Dachüberhänge, Verschattung von Dächern mittels Anlagen für solare Energiegewinnung).

- Technische bauliche Maßnahmen wie Belüftungstechnik, Wärme-/Kältetauscher, Raumventilatoren, evtl. auch Einsatz von Klimaanlagen in besonders sensiblen Bereichen.

- Hitzeadäquate Gebäudeplanung bei Neubauten (Berücksichtigung u.a. der Architektur, des Breiten-/Höhenverhältnisses, der Bebauung entlang von Straßen sowie der Ausrichtung und Lage) im städtischen wie auch im ländlichen Raum.

- Verwendung von hitzereduzierenden und Vermeidung von hitzespeichernden Baumaterialien.

- Trinkwasserspender in Gebäuden und im öffentlichen Raum installieren.

- Einrichtung und Nutzung sogenannter „Cooling Centres“, z. B. öffentliche, kühle Räumlichkeiten, z. B. in Behörden, Einkaufspassagen, Kirchengebäuden, Büchereien und Bahnhöfen.

Stadt- und bauplanerische Maßnahmen:

- Erhalt oder Schaffung von schattenspendenden Grünanlagen und Parks bestenfalls mit kühlenden Verdunstungsflächen wie Wasserflächen oder -spielen.

- Einrichten von großzügigen Schattenplätzen (baulich mittels Pavillons, Außendächern, Markisen, feststehenden Sonnenschirmen oder Sonnensegeln, durch Neuanpflanzungen oder den Erhalt von Baumbeständen mit dichten Blattkronen).

- Einrichten von Befeuchtungsanlagen in Außenanlagen und für Terrassen.
- Hitzereduktion durch Freihalten bzw. Schaffen von Luftleitbahnen und Kaltluftentstehungsgebieten.

- Reduzierung des Versiegelungsgrades von offenen und öffentlichen Plätzen zur Vermeidung der Entstehung von hohen Hitze- und UV-Belastungen aufgrund von Reflexion.

- Förderung von Baum- und Buschpflanzungen und Dachbegrünungen (hier auf allergenarme und hitze-/trockentolerante Pflanzen achten).

- Einrichten von schattenspendenden Vordächern und Überdachungen, vorzugsweise mit Materialien, die auch die Belastung durch UV-Strahlung reduzieren.

- Einrichtung von fest installierten Trinkwasserspendern im öffentlichen Raum.

Weitere Hinweise sind zu finden auf den Internetseiten des Kompetenzzentrums Klimafolgen und Anpassung (KomPass) unter http://www.stadtklimalotse.net/ massnahmenkatalog/.

\section{Handlungsempfehlung zu Kernelement VIII}

\section{Monitoring und Evaluierung der Maßnahmen}

Die WHO empfiehlt eine zeitnahe epidemiologische Beobachtung und die Evaluation der Hitzeschutzmaßnahmen.

Monitoringsysteme und Evaluierungen haben die Aufgabe, Hitzeereignisse und deren Folgen quantitativ zu erfassen und zu bewerten, um gegebenenfalls Nachbesserungen und Weiterentwicklungen der Interventionsmaßnahmen zu veranlassen. Sie sind zur Implementierung erster wichtiger Anpassungsmaßnahmen zunächst nicht erforderlich, langfristig jedoch sehr sinnvoll. Keinesfalls sollten Maßnahmen - gleich welcher Art - aufgrund von Monitoring-Schwierigkeiten nicht geplant und durchgeführt werden.

1. Monitoring: Um die gesundheitlichen Auswirkungen einer Hitzeperiode wirkungsvoll zu überwachen, müssen die Daten zeitnah zur Verfügung stehen. Dies dient dazu, Anpassungen an Hitzeperioden und Gegenmaßnahmen einschließlich einer besseren In- formation der Bevölkerung ergreifen zu können.

Des Weiteren kann auf diese Weise die Wirksamkeit von Interventionsmaßnahmen bewertet werden.

Die für diese zeitnahen Auswertungen erforderlichen Daten stammen z.B. aus folgenden Quellen:

- Notaufnahmen und Aufnahmeregister der Krankenhäuser

- Rettungsdienste

- Ärztliche Bereitschaftsdienste

- Notfalltelefone

- Standesämter, Statistische Landesämter (Anzahl Todesfälle)

2. Wichtig ist, dass überhaupt $\mathrm{Da}$ ten erhoben werden. Es sollte eine Erhebung tagesbezogener Morbiditäts- und Mortalitätsfälle für die spätere Untersuchung von Expositions-Effektbeziehungen erfolgen, auch unter Berücksichtigung kombinierter Gesundheitswirkungen von sommerlicher Hitze und verstärkter Verunreinigung der Luft.

Die Informations- und Datensammlung könnte aus folgenden Quellen erfolgen:

- Notaufnahmen und Aufnahmeregister der Krankenhäuser

- Notdienste

- Krankenkassen

- Gesundheitsämter, Standesämter, Statistische Landesämter

3. Die Maßnahmen des Hitzeaktionsplans sollten evaluiert werden, damit der Gesundheitsschutz der Bevölkerung weiter verbessert werden kann. Hierfür ist es erforderlich, die tatsächlich erfolgten Maßnahmen zu dokumentieren.

4. Es sollte geprüft werden, ob und wie die Auswertung und Nutzung der Daten auf verschiedenen Ebenen des öffentlichen Gesundheitswesens erfolgen kann (z. B. Gesundheitsämter, Landesgesundheitsbehörden, Robert Koch-Institut).

\section{Fazit}

Hitzeaktionspläne sind von Ländern bzw. Kommunen individuell zu erstellen, da jeweils die örtlichen Gegebenheiten und hier insbesondere die klimatischen Bedingungen die Basis für die 


\section{Infobox 2 Ein Beispiel zur Umsetzung aus der Praxis}

Einzelne Ansätze zur Aktionsplanung bei Hitzeereignissen gibt es in Deutschland bereits. Im Bundesland Hessen wurde beispielsweise als Reaktion auf den Hitzesommer 2003 eine Arbeitsgruppe zur Gesundheitsprävention bei Hitzeperioden eingerichtet, die Maßnahmenempfehlungen für die Pflege (siehe Weiterführende Literatur) oder allgemeine Verhaltenstipps für Hessen erarbeiteten. Teilgenommen haben unter anderem das hessische Sozialministerium, die Ebene der Regierungspräsidien, einige kommunale Gesundheitsämter, der DWD und Vertreter des medizinischen Dienstes der Krankenversicherung, der AOK und der kassenärztlichen Vereinigung. Für eine nachhaltige Hitzeaktionsplanung müsste ein entsprechend ausgerichtetes und zusammengesetztes Fachgremium gemäß den Empfehlungen zum Kernelement I eingerichtet und verstetigt werden. Zurückgehend auf ein Ergebnis aus der vorgenannten Arbeitsgruppe wurde in Hessen eine weitere Warnstufe etabliert. So wird bei Hitzeperioden die sogenannte Warnstufe 2 ausgerufen, wenn der DWD vor extremer Hitzebelastung warnt oder für mindestens drei Tage in Folge eine starke Hitzebelastung besteht. Bei Erreichen der Warnstufe 2 gibt das Hessische Ministerium für Soziales und Integration eine Pressemitteilung heraus (siehe https://soziales.hessen.de/presse/ pressemitteilung/hitzewarnstufe-2-wirdmorgen-erreicht), die nicht nur die stationäre Pflege, sondern die Allgemeinbevölkerung einschließlich der Pflegebedürftigen und älteren Menschen anspricht. Auch wird dabei auf Tipps zum Verhalten bei extremer Hitze und zu Sonnenschutzmaßnahmen verwiesen.

Entwicklung und Umsetzung geeigneter und sinnvoller Maßnahmen darstellen. Die vorliegenden Empfehlungen sollen Hilfe bei der Erstellung solch angepasster Hitzeaktionspläne sein. Zusätzlich zu diesen Handlungsempfehlungen dienen auch Anregungen aus der Praxis (• Infobox 2).

Die vorliegenden Handlungsempfehlungen umfassen sowohl kurzfristig umsetzbare Aktivitäten (z. B. Einrichten von Kommunikationswegen, Handlungsfeld III) als auch erst längerfristig umsetzbare Aktivitäten (z. B. Stadtplanerische Maßnahmen, Handlungsfeld VII). Während in einigen Fällen die Implementierung schon weit fortgeschritten ist (z.B. Etablierung des Hitzewarnsystems, Handlungsfeld II), geben andere Aktivitäten noch viel Raum für Entwicklung und stellen vor allem eine Herausforderung für die Zukunft dar (z. B. Einrichtung eines zeitnahen Monitorings, Handlungsfeld VIII).

Jede zuständige Behörde soll mit diesen Empfehlungen ermutigt werden, selbst bei geringen personellen und finanziellen Ressourcen, die Entwicklung eines Hitzeaktionsplans mit den verfügbaren „Bordmitteln“ voranzubringen, um für den nächsten Hitzesommer besser gewappnet zu sein. Viele der Maßnahmen können kostengünstig umgesetzt werden, da es im Wesentlichen um die Bewusstseinsschärfung für diese Thematik geht, um hierdurch Änderungen von Verhaltensweisen bei den Betroffenen zu bewirken.

Auch bauliche Maßnahmen können mitunter mit relativ geringem Aufwand durchgeführt werden, z. B. bei ohnehin notwendigen Änderungen an bestehenden Bauten, bei Stadtplanungen oder bei Bauvorhaben.

Wichtig ist hierfür ein Konsens aller Beteiligten: Das Ziel ist, die Gesundheit der Bevölkerung vor den negativen Folgen extremer Hitze zu schützen.

\section{Autorenteam}

\section{Leitung}

Dr. Wolfgang Straff, Umweltbundesamt Dr. Hans-Guido Mücke, Umweltbundesamt

\section{Mitglieder}

Regine Baeker, Ministerium für Arbeit, Soziales, Gesundheit, Frauen und Familie, Brandenburg

Dr. Cornelia Baldermann, Bundesamt für Strahlenschutz

Dr. Angela Braubach, Bundesamt für Bevölkerungsschutz und Katastrophenhilfe Dr. Jutta Litvinovitch, Bundesministerium für Umwelt, Naturschutz, Bau und Reaktorsicherheit

Prof. Dr. Andreas Matzarakis, Deutscher Wetterdienst

Gudrun Petzold, Ministerium für Soziales, Gesundheit, Wissenschaft und Gleichstellung, Schleswig-Holstein

Dr. Ute Rexroth, Robert Koch-Institut Susanne Schroth, Landesamt für Naturschutz, Umwelt und Geologie, Hessen
Norbert Stutzinger-Schwarz, Bundesministerium für Umwelt, Naturschutz, Bau und Reaktorsicherheit

Die vorliegende Fassung dieser Handlungsempfehlungen wurde der Bund/ Länder Ad-hoc Arbeitsgruppe ,Gesundheitliche Anpassung an die Folgen des Klimawandels (GAK)` bei der letzten Sitzung am 22. und 23. März 2017 abschließend vorgestellt, diskutiert und von ihr beschlossen sowie der Veröffentlichung zugestimmt.

Open Access. Dieser Artikelwird unter der Creative CommonsNamensnennung 4.0 International Lizenz (http://creativecommons.org/ licenses/by/4.0/deed.de) veröffentlicht,welche die Nutzung, Vervielfältigung, Bearbeitung, Verbreitung undWiedergabe in jeglichemMedium und Format erlaubt, sofern Sie den/die ursprünglichen Autor(en) und die Quelle ordnungsgemäßnennen, einen Link zurCreativeCommons Lizenz beifügen und angeben, obÄnderungen vorgenommenwurden.

\section{Literatur}

1. Bundesregierung (2008) Deutsche Anpassungsstrategie an den Klimawandel. In: Deutscher Bundestag Drucksache 16/11595. Bundesanzeiger Verlagsgesellschaft mbH, Köln

2. Kandarr J, Reckert H, Mücke H-G (2014) Anpassung an die gesundheitlichen Risiken des Klimawandels als Aufgabe des umweltbezogenen Gesundheitsschutzes. Bundesgesundheitsblatt Gesundheitsforschung Gesundheitsschutz 57:1209-1215

3. Zielo B (2016) Die Auswirkungen des Klimawandels auf die menschliche Gesundheit am Beispiel von Hitzewellen als Extremwetterereignis. In: Fakultät für Umwelt und Natürliche Ressourcen. Albert-Ludwigs-Universität, Freiburg

4. Zielo B, Matzarakis A (2017) Bedeutung von Hitzeaktionsplänen für den präventiven Gesundheitsschutz in Deutschland. Gesundheitswes. unveröffentlichter Beitrag (akzeptiert)

5. Umweltbundesamt, Robert Koch-Institut (2013) Klimawandel und Gesundheit - Allgemeiner Rahmen zu Handlungsempfehlungen für Behörden und weitere Akteure in Deutschland. Umweltbundesamt und Robert-Koch Institut, Berlin, S 34

6. Kovats S, Jendritzky G (2006) Heat-waves and human health. In: Menne B, Ebi KL (Hrsg) Climate change and adaptation strategies for human health. Steinkopff, Darmstadt, S 63-97

7. Jendritzky G, Koppe C (2006) Die Auswirkungen von thermischen Belastungen auf die Mortalität. In: Lozán J (Hrsg) Warnsignal Klima - Gesundheitsrisiken. Wissenschaftliche Auswertungen, Hamburg, S 149-153

8. EEA (2012) Impacts of Europe's changing climate 2008 indicator-based assessment. In: EEA Report No 4/2008.

9. Barriopedro D, Fischer EM, Luterbacher J, Trigo RM, García-Herrera R (2011) The hot summer of 2010. Redrawing Temp Rec Map Eur Sci 332:220-224 


\section{Weiterführende Literatur, die für die Erarbeitung regionaler Hitzeaktionspläne hilfreich sein kann}

\section{WHO-Veröffentlichungen}

Heat Health Action Plan, WHO Regional Office for Europe, 2008 http://www.euro.who.int/_data/ assets/pdf_file/0006/95919/E91347.pdf

Public health advice on preventing health effects of heat. New and updated information for different audiences, 2011

http://www.euro.who.int/_data/assets/pdf_file/0007/147265/Heat_information_

sheet.pdf?ua=1

Improving public health responses to extreme weather/heat-waves - EuroHEAT, 2009

http://www.euro.who.int/_data/assets/pdf_file/0010/95914/E92474.pdf?ua=1

\section{Deutschsprachige Veröffentlichungen}

Umweltbundesamt und Deutscher Wetterdienst (2008):

Ratgeber: Klimawandel und Gesundheit. Informationen zu gesundheitlichen Auswirkungen sommerlicher Hitze, Hitzewellen und Tipps zum vorbeugenden Gesundheitsschutz

https://www.umweltbundesamt.de/publikationen/ratgeber-klimawandel-gesundheit

Umweltbundesamt und Robert Koch-Institut (2013):

Klimawandel und Gesundheit - Allgemeiner Rahmen zu Handlungsempfehlungen für Behörden und weitere Akteure in Deutschland. http://www.apug.de/archiv/pdf/klimawandel/allgemeiner_rahmen_zu_handlungsempfehlungen_klimawandel_und_gesundheit_final.pdf

Capellaro M, Sturm D (2015):

Evaluation von Informationssystemen zu Klimawandel und Gesundheit

Band 1: Anpassung an den Klimawandel: Evaluation bestehender nationaler Informationssysteme (UV-Index, Hitzewarnsystem, Pollenflug- und Ozonvorhersage) aus gesundheitlicher Sicht - Wie erreichen wir die empfindlichen Bevölkerungsgruppen? Umweltbundesamt (Hrsg.). Umwelt und Gesundheit 03/2015.

Band 2: Anpassung an den Klimawandel: Strategie für die Versorgung bei Extremwetterereignissen. Umweltbundesamt (Hrsg.). Umwelt und Gesundheit 04/2015.

Erhältlich unter www.umweltbundesamt.de

Regierungspräsidium Gießen - Hessische Heimaufsicht (2009):

Außergewöhnliche Hitzeperioden: Vorbereitung und Vorgehen stationärer Pflegeeinrichtungen https://rp-giessen.hessen.de/sites/rp-giessen.hessen.de/files/content-downloads/Handlungsempfehlung_Hitze_0.pdf)

Hochschule Fulda (2012):

Hessischer Aktionsplan zur Vermeidung hitzebedingter Gesundheitsbeeinträchtigungen der Bevölkerung (HEAT) http://www.hlnug.de/fileadmin/dokumente/klima/inklim_a/heat.pdf

Bundesministerium für Umwelt, Naturschutz und Reaktorsicherheit (2010):

Klimawandel, Extremwetterereignisse und Gesundheit. Bericht der Internationalen Fachkonferenz vom 29./30. November 2010. http://www.bmub.bund.de/fileadmin/Daten_BMU/Pools/Broschueren/klimawandel_extremwetter_konferenzbericht_bf.pdf

10. Tröltzsch J, Görlach B, Lückge H, Peter M, Sartorius $C$ (2011) Ökonomische Aspekte der Anpassung an den Klimawandel - Literaturauswertung zu Kosten und Nutzen von Anpassungsmaßnahmen an den Klimawandel. Umweltbundesamt, Dessau

11. Eis D, Helm D, Laußmann D, Stark K (2010) Klimawandel und Gesundheit - Ein Sachstandsbericht. Robert Koch-Institut, Berlin

12. Matthies F, Bickler G, Marin N, Hales S (2008) HeatHealth Action Plans. Regional Office for Europe, Copenhagen

13. Mücke H-G, StraffW, Faber M et al (2013) Klimawandel und Gesundheit: Allgemeiner Rahmen zu Handlungsempfehlungen für Behörden und weitere Akteure in Deutschland. Robert Koch-Institut und Umweltbundesamt, Berlin, $\mathrm{S} 34$

14. Grobe TG, Heller G, Szecsenyi J (2014) Arztreport Schwerpunkt: Hautkrebs. Barmer GEK, Siegburg

15. Gesellschaft der Epidemiologischen Krebsregister in Deutschland e. V. (2016) Atlas der Krebsinzidenz und -mortalität in Deutschland (GEKID-Atlas)
http://www.gekid.de/Atlas/CurrentVersion/atlas. html. Zugegriffen: 2. Mai 2017

16. Arbeitsgemeinschaft der Wissenschaftlichen Medizinischen Fachgesellschaften e. V. (2014) S3Leitlinie Prävention von Hautkrebs, Langversion 1.0. In: Leitlinienprogramm Onkologie. Deutsche Krebsgesellschaft, Deutsche Krebshilfe

17. Grewe HA (2012) Hessischer Aktionsplan zur Vermeidung hitzebedingter Gesundheitsbeeinträchtigungen der Bevölkerung (HEAT). Hochschule Fulda - Fachbereich Pflege und Gesundheit, Fulda

18. Matzarakis A (2016) Das Hitzewarnsystem des Deutschen Wetterdienstes (DWD) und seine Relevanz für die menschliche Gesundheit. Gefahrstoffe Reinhaltung der Luft Bd. 76. Springer-VDI-Verlag, Düsseldorf 